the project and to Dr. J. M. Hirst, Rothamsted Experimental Station, for many helpful suggestions.

West African Maize Rust Research Unit,

Department of Agricultural Research, Moor Plantation, Ibadan, Nigeria. July 27.

1 Hirst, J. M., Ann. App. Biol., 39, 257 (1952),

2 Hirst, J. M., Trans. Brit. Myc. Soc., 36, Part 4, 375 (1953). ${ }^{3}$ Keay, R. W. J., "An Outline of Nigerian Vegetation", pp. 14-23
(Gov. Printer, Nigeria; Crown Agents, London, 1953).

+ Brooks, C. E. P., Quart. J. Roy. Met. Soe. London, 42 (1916) ; 46 (1.920).

${ }^{5}$ Gregory, P. H., Ann. App. Biol., 38, 357 (1951).

\section{A Statistical Information Theory of Visual Thresholds}

Ir is well known that, for constant stimulus duration, the visual threshold intensity, $I$, is a function of the stimulus area $A$. In the case of absolute thresholds it has been generally accepted that: (i) for areas up to about $6^{\prime}, A I=$ constant (Ricco's law); (ii) for areas between about $6^{\prime}$ and perhaps $20^{\circ}, A I^{2}=$ constant (Piper's law).

It seems reasonable to regard the absolute threshold as a special case of the differential threshold. It is then important to discover whether these relations in fact hold for the differential case. Brindley ${ }^{1}$ has shown that Piper's law holds for differential thresholds where the test field is discriminated against a large field of constant area. Ricco's law is also found to hold for blue light, optical effects making it difficult to test for other colours. Piper's law for differential threshold where the background area is large and fixed has been formulated:

$$
\frac{\Delta I}{I}=\text { const. } \sqrt{\frac{1}{A}}
$$

We find, by using various small background areas, that the more general case where either area is variable may be written, at least approximately,

$$
\frac{\Delta I}{I}=\text { const. } \sqrt{\left(\frac{1}{A_{1}}+\frac{1}{A_{2}}\right)}+C
$$

This would give the Weber-Fechner law, $\Delta I / I=C$, for very large areas $A_{1}$ and $A_{2}$, which would make $\left(\frac{1}{A_{1}}+\frac{1}{A_{2}}\right)$ negligibly small.

We also find that this may be further generalized to cover low intensities in the region of breakdown of the Weber-Fechner law by adding a small constant thus :

$$
\frac{\Delta I}{I+k}=\text { const. } \sqrt{\frac{1}{A_{1}}+\frac{1}{A_{2}}}+C
$$

The value of $k$ in terms of equivalent intensity we estimate at about $0.04 \mathrm{ft}$. l. For the absolute threshold, when $I=0$, we should then have $\Delta I / k=C$ when the areas are large.

It appears that these, and perhaps other threshold and summation phenomena, may be described in terms of statistical information available in neural information channels. If we suppose that the area $A$ inspected is to be regarded as a set of $n$ independent observations, where $n$ is approximately proportional to $A$, and that each of these observations is subject to random variation through noise in the system, then for $\Delta I$ to be detected against $I$, in some fixed proportion of the number of trials (for example, an 80 per cent experimental criterion), we must have

$$
\frac{[(r+\Delta r)-r]-C}{\sqrt{\left(\frac{1}{A_{1}}+\frac{1}{A_{2}}\right) r}}=\text { constant }
$$

where $(r+\Delta r), r$, are the mean neural pulse-rates corresponding to intensities $I+\Delta I, I$, and $V$ is the variance of neural pulse-rate over the sample.

We have introduced the constant $C$ to explain the fact that few false positives occur in threshold experiments. Without some criterion of difference greater than zero, we should expect about 50 per cent false positives. The proportion of false positives may change with the observer's attitude, or 'set', indicating that $C$ may vary within limits. It is tempting to regard $k$ as arising from the internal noise present in the system, and to think of the absolute threshold as the case where the signal is to be distinguished against noise background.

If, as seems possible from electrophysiological data, $r$ is proportional to $\log (I+k)$, (4) reduces to (3) above for $\Delta I$ somewhat less than $I+k$, and we expect this to hold for relatively small values of $\Delta I$ even when $I=0$, that is, for absolute thresholds. In the latter case, as the test area diminishes, however, and the value of $\Delta I$ consequently increases, the approximation $\Delta I / k$ used for $[(r+\Delta r)-r]$ is no longer valid, and the more accurate expression $[\log (k+\Delta I)-\log k]$ is required. For $A_{2}$ large, (4) can then be written

$$
1+\frac{\Delta I}{k}=\exp (C+\alpha \sqrt{1 / A)},
$$

writing $A$ for $A_{1}$ and denoting the constant involved by $\alpha$.

For very large $A$, the terms containing $A$ are negligible; therefore

$$
1+\frac{\Delta I}{k}=\text { const., or } \Delta I=\text { const. }
$$

For smaller $A$ :

$$
1+\frac{\Delta I}{k}=\exp C(1+\alpha / \sqrt{A})
$$

or

$$
\sqrt{A} \Delta I=\text { const. }+ \text { const. } \sqrt{A}
$$

For even smaller $A$ :

$$
1+\frac{\Delta I}{k}=\exp C\left(1+\sqrt{\frac{\alpha}{\bar{A}}}+\frac{\alpha^{2}}{2 A}+\ldots\right)
$$

It thus appears that Riceo's and Piper's law's might be regarded as the different approximations to the logarithmic equation which can be used at different levels. The expression (7) appears to fit Willmer's ${ }^{2}$ data satisfactorily. We are undertaking experiments to determine the various constants involved.

Mr. R. T. Leslie, Mr. E. R. F. W. Crossman and Dr. G. S. Brindley have been most helpful in discussing these and related ideas.

Psychological Laboratory,

R. L. GREGORY Downing Place, Cambridge.

Medical Research Council Applied Psychology Unit, 15 Chaucer Road,

Cambridge. Aug. 26.

1 Brindley, G. S., J. Physiol., 124, 400 (1954).

"Willmer, E. N., Quart. J. Exp. Psychol., 2, Part 2 (May 1950). 